\title{
Acanthamoeba keratitis related to contact lens use in a tertiary hospital in China
}

Weiwei $\mathrm{Li}^{1+}$, Zhiqun Wang ${ }^{2 \dagger}$, Jinghao Qu', Yang Zhang ${ }^{2}$ and Xuguang Sun²*

\begin{abstract}
Background: To report the clinical and microbiological features of Acanthamoeba keratitis (AK) related to contact lens use in a tertiary hospital in China.

Methods: In this retrospective study, the medical results of 61 cases of AK related to contact lens use from January 2000 to December 2017 were reviewed. The data included patients' demographics, lens type, history, risk factors, disease stages, corneal scraping and culture reports, and treatments. Moreover, genotypic identification of some of the isolates was carried out with a PCR assay and sequence analysis of the $18 \mathrm{~S}$ ribosomal DNA gene.

Results: There were 64 eyes included in the study. A total of $32.8 \%$ of the patients wore soft contact lenses, and $67.2 \%$ of patients used overnight orthokeratology. In the cases (20 eyes) in the early stage, $65 \%$ (13 eyes) had positive results according to Giemsa-stained smears, and $0.9 \%$ sodium chloride $(\mathrm{NaCl})$ wet mounts revealed trophozoites in 7 eyes (35\%). Six eyes (30\%) were diagnosed by confocal microscopy combined with clinical signs. In the orthokeratology patients, 87.8\% (36/41) rinsed their lenses and/or cases with tap water; 55\% of soft-lens wearers wore their lenses while showering. The genotype of 9 isolates was determined, and all the strains belonged to genotype T4. In the orthokeratology group, the number of patients who required therapeutic penetrating keratoplasty after 2005 was less than that before 2005 (chi-square test, $X^{2}=4.209, P=0.04$ ).

Conclusions: More than two-thirds of the cases were associated with orthokeratology. Examinations with Giemsastained smears, $0.9 \% \mathrm{NaCl}$ wet mounts and confocal microscopy should be performed for patients who are highly suspected of having early-stage AK to help with early diagnosis. In the orthokeratology group, the rate of therapeutic keratoplasty after 2005 was less than that before 2005.
\end{abstract}

Keywords: Acanthamoeba keratitis, Contact lens, Genotype

\section{Background}

Acanthamoeba keratitis (AK) is a severe and visionthreatening infection of the cornea. In the 1980s, AK dramatically increased in conjunction with the increasing use of soft contact lenses (SCLs) [1]. AK occurred most frequently in contact lens wearers in developed countries $[2,3]$. In recent years, the incidence has increased $[4,5]$. AK cases related to contact lenses have also been reported in other countries [6,7]. Although contact lens use is not the most common risk factor in

\footnotetext{
* Correspondence: sunxg2010@163.com

${ }^{+}$Weiwei Li and Zhiqun Wang contributed equally to this work.

${ }^{2}$ Beijing Institute of Ophthalmology, Beijing Tongren Eye Center, Beijing

Tongren Hospital, Beijing Ophthalmology \& Visual Sciences Key Laboratory,

Capital Medical University, Address: 17 Hou Gou Lane, Chong Nei Street,

Beijing 100005, China

Full list of author information is available at the end of the article
}

China [8], AK continues to be seen in contact lens wearers, especially users of orthokeratology.

A good prognosis of AK depends on an early diagnosis, but early diagnosis remains a challenge. Several methods such as corneal scraping and confocal microscopy are fast and useful for diagnosis in the early stages. In some cases of early-stage AK, trophozoites were active, and the number of cysts was small. Diagnosis by in vivo confocal microscopy may be difficult, and direct examination of trophozoites by $0.9 \%$ sodium chloride $(\mathrm{NaCl})$ may be helpful. In this retrospective study, the clinical and microbiological data of AK related to contact lens use were analysed in a tertiary hospital in Beijing, China.

(C) The Author(s). 2019 Open Access This article is distributed under the terms of the Creative Commons Attribution 4.0 International License (http://creativecommons.org/licenses/by/4.0/), which permits unrestricted use, distribution, and reproduction in any medium, provided you give appropriate credit to the original author(s) and the source, provide a link to the Creative Commons license, and indicate if changes were made. The Creative Commons Public Domain Dedication waiver (http://creativecommons.org/publicdomain/zero/1.0/) applies to the data made available in this article, unless otherwise stated. 


\section{Methods}

A retrospective review of the medical records of patients diagnosed with AK associated with contact lens use from January 2000 to December 2017 at Beijing Tongren Hospital was performed. This study was approved by the Institutional Review Board of Beijing Tongren Hospital and adhered to the tenets of the Declaration of Helsinki.

Cases were included in this study if corneal scraping and/or culture were positive for Acanthamoeba. The corneal samples were Giemsa stained for the detection of cysts. The material obtained in the next scraping sample was combined with $0.9 \% \mathrm{NaCl}$ on a clean sterile glass slide as a wet mount to detect the trophozoites by direct microscopy (Olympus BX51, Tokyo, Japan); the cysts could also be detected. The images were captured using a colour digital camera (DP-12, Olympus). The samples were streaked onto non-nutrient agar plates covered with Escherichia coli and incubated at $28^{\circ} \mathrm{C}$ for 5 to 15 days for the culture of amoebae. When corneal scraping and culture results were negative, cases were identified if Acanthamoeba cysts were observed by in vivo confocal microscopy (Heidelberg Engineering GmbH, Dossenheim, Germany) and typical clinical signs were present. The following data were collected: demographic information, lens type, history, risk factors, disease stages, and simultaneous bacteria and fungi culture reports. PCR was performed to determine the genotype of the Acanthamoeba isolates. The DNA extraction and PCR assays were performed as described in our previous study [9]. The PCR assay was performed with the genus-specific primers JDP1 (5'-GGCCCAGATC GTTTACCGTGAA) and JDP2 (5'- TCTCACAAGC TGCTAGGGGAGTCA). Then, direct sequencing of the PCR products was performed with the conserved primer 892C (5'-GTCAGAGGTGAAATTCTTGG).

Treatments were also analysed. The disease stages (Table 1) and treatments referred to our previous study [8]. Topical anti-amoebic therapy included chlorhexidine and/or polyhexamethylene biguanide (PHMB). The concentrations of these drugs were determined according to the disease stage, and oral itraconazole was administered to some patients. If the infectious process spread despite aggressive medical therapy or the case of AK was severe, therapeutic keratoplasty was performed. If the patients were coinfected with Acanthamoeba and other pathogens, appropriate treatment was administered simultaneously.

Statistical analyses were performed using the SPSS statistical software package (SPSS for Windows, version 17.0; SPSS, Inc., Chicago, IL). Independent-sample ttests and chi-square tests were conducted to compare the differences in the clinical data. A $P$ value less than 0.05 was considered statistically significant.

\section{Results}

There were 61 patients (64 eyes) included in the study; 24 (39.3\%) patients were male and 37 (60.7\%) patients were female. The age varied between 9 and 48 years old (mean $19.95 \pm 7.06$ years old). Of the 61 patients, 20 patients (32.8\%) wore SCLs, and $41(67.2 \%)$ patients used overnight orthokeratology. There were 3 bilateral cases in this study, and all of these patients used orthokeratology. The cases in each year are shown in Fig. 1 (Additional file 1); the incidence peaked in 2001.

Table 2 shows the demographic characteristics, clinical features, microbiological culture results and treatments for all the cases. There was no significant difference in gender between the SCL and orthokeratology groups (chi-square test, $X^{2}=0.005, P=0.942$ ). The orthokeratology wearers were younger than the patients who wore SCLs (independent-sample t-test, $t=4.338, P=0.000$ ). The symptom duration ranged from 5 days to 3 months. Fifty-four eyes (84.4\%) had positive results according to the Giemsa-stained smears. In addition, trophozoites were detected in 19 eyes $(29.7 \%)$ by $0.9 \% \mathrm{NaCl}$ wet mounts. When the $0.9 \% \mathrm{NaCl}$ wet mount was directly examined, the trophozoite had an oval or elongated outline, a large central nucleolus and hyaline pseudopodia (Fig. 2); it moved slowly with its pseudopodia in the tissue. In early-stage cases (20 eyes), 65\% (13 eyes) had positive results according to the Giemsa-stained smears, and trophozoites were seen in 7 eyes $(35 \%)$ on the $0.9 \%$ $\mathrm{NaCl}$ wet mounts. Six eyes (30\%) were diagnosed by confocal microscopy combined with clinical signs. The culture-positive rate of Acanthamoeba was 76.6\% (49/64). Six cases $(9.84 \%)$ had polymicrobial infection, and most $(5 / 6)$ were soft-contact lens wearers. The isolated organisms were Pseudomonas aeruginosa and Staphylococcus epidermidis. No fungal organisms were isolated. The genotypes of 9 isolates from nine patients were identified.

Table 1 The disease stages [8]

\begin{tabular}{ll}
\hline Stage & Manifestation \\
\hline Early stage & $\begin{array}{l}\text { Pseudodendritic or punctate corneal epithelial lesions, dot infiltrations under the epithelium, recurrent epithelial } \\
\text { erosion, radial corneal neuritis, corneal ulcer smaller than } 4 \mathrm{~mm} \text { in diameter in the anterior stroma }\end{array}$ \\
Advanced stage & Deep stromal ulcer greater than $5 \mathrm{~mm}$ and less than $8 \mathrm{~mm}$ in diameter \\
Late stage & The deep stromal ulcer greater than $8 \mathrm{~mm}$ in diameter and hypopyon, central corneal thinning and perforation
\end{tabular}




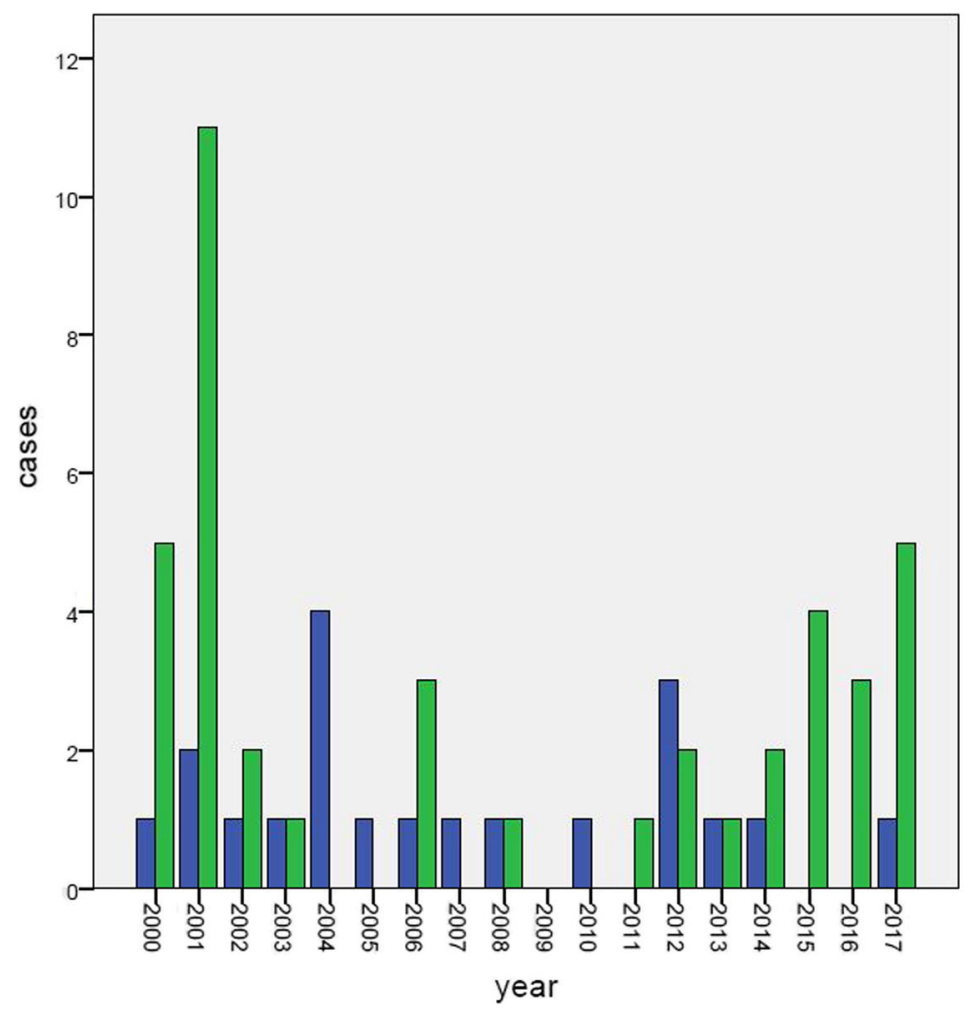

soft contact lens

orthokeratology

Fig. 1 Cases of Acanthamoeba keratitis related to contact lens use in each year

Four patients were male, and 5 patients were female. Six isolates were from soft-contact lens wearers, and 3 isolates were from patients who used orthokeratology lenses. All of the Acanthamoeba strains belonged to genotype T4. Among the 9 strains, two were identified as T4/6, and the

Table 2 Results of demographic characteristic, clinical feature, microbiological culture and the treatment in each lens type

\begin{tabular}{lll}
\hline Characteristic & $\begin{array}{l}\text { Soft contact lens } \\
(n=20, \text { eyes }=20)\end{array}$ & $\begin{array}{l}\text { Orthokeratology } \\
(n=41, \text { eyes }=44)\end{array}$ \\
\hline $\begin{array}{l}\text { Gender, } \mathrm{n}(\%) \\
\text { Female }\end{array}$ & $12(60.0)$ & $25(61.0)$ \\
male & $8(40.0)$ & $16(39.0)$ \\
Age (years) & $25.90 \pm 8.81$ & $17.05 \pm 3.41$ \\
$\begin{array}{l}\text { Positive result in culture for } \\
\text { Acanthamoeba, eyes(\%) }\end{array}$ & $16(80.0)$ & $33(75.0)$ \\
$\begin{array}{l}\text { Positive result in culture for } \\
\text { bacteria, eyes(\%) }\end{array}$ & $5(25.0)$ & $1(2.3)$ \\
$\quad \begin{array}{l}\text { Pseudomonas aeruginosa } \\
\quad \text { Staphylococcus epidermidis }\end{array}$ & $3(15)$ & $0(0)$ \\
Treatment, eyes (\%) & $2(10)$ & $1(2.3)$ \\
$\quad \begin{array}{l}\text { Medical therapy } \\
\text { Therapeutic penetrating } \\
\text { keratoplasty }\end{array}$ & $4(20.0)$ & $33(75.0)$ \\
\hline
\end{tabular}

others were identified as T4/8, T4/9, T4/13, T4/24, T4/31, $\mathrm{T} 4 / 34$, and $\mathrm{T} 4 / 41$, respectively.

The different disease stages according to each lens type are summarized in Table 3. A total of $76.6 \%$ of the eyes were cured of corneal scars by medical therapy, and the remainder of the eyes underwent therapeutic keratoplasty. There was no recurrence after surgery. In the orthokeratology group, the number of patients who required therapeutic penetrating keratoplasty after 2005 $(3 / 22)$ was less than that before 2005 (8/19) (chi-square test, $X^{2}=4.209, P=0.04$ ).

In the orthokeratology group, $87.8 \%(36 / 41)$ of the patients rinsed their lenses and/or cases with tap water, and in the SCL group, 11 patients (55\%) wore their lenses while showering (5 cases, $25.0 \%$ ) or sleeping (6 cases, 30.0\%).

\section{Discussion}

The Acanthamoeba genus is a free-living amoebae that can be found in the air, soil and water. In China, the most common risk factor is ocular trauma, followed by contact lens wear [8]; however, cases related to contact lens use were seen almost yearly in this study. In addition, a notable finding of our previous study was that the number of cases of AK was nearly one-half the number of cases of microbial keratitis related to contact 


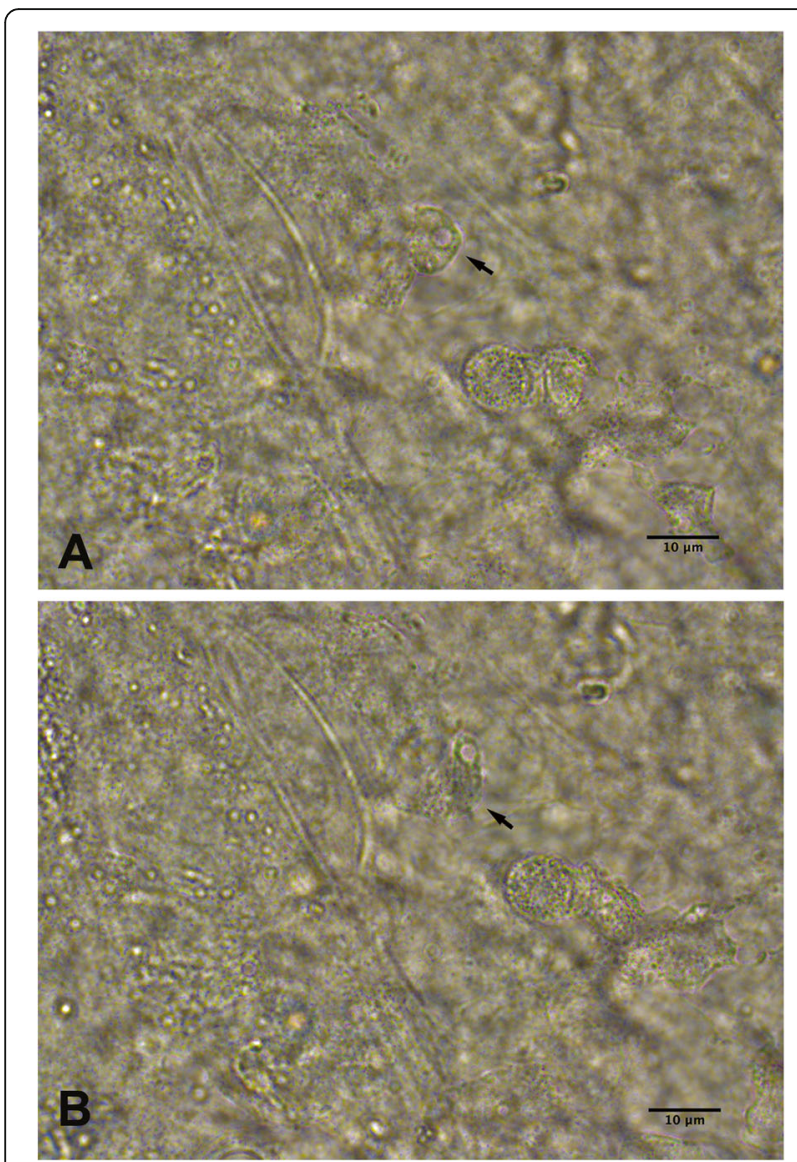

Fig. 2 Acanthamoeba trophozoite in a 0.9\% sodium chloride wet mount (black arrow) a The trophozoite was oval with a large nucleolus. $\mathbf{b}$ The same Acanthamoeba trophozoite became elongated in shape after several seconds

lens wear [10]. However, few studies have reported the clinical and microbiological features of contact lensrelated AK in China.

In this study, the peak year of occurrence was 2001, which may be due to the outbreak of AK associated with orthokeratology in 2001 [11, 12]. At that time, the government intervened to regulate the orthokeratology market, and the number of patients fitted with orthokeratology decreased. The cases of AK related to orthokeratology decreased correspondingly [13]. In this study, there were no patients with cases related to orthokeratology in 2004 and 2005. However, in recent years, cases have reemerged and increased slightly. This trend has also been seen in other countries. It was reported that

Table 3 The cases in each lens type with different disease stages

\begin{tabular}{llll}
\hline Lens type & $\begin{array}{l}\text { Early stage, } \\
\text { eyes(\%) }\end{array}$ & $\begin{array}{l}\text { Advanced stage, } \\
\text { eyes(\%) }\end{array}$ & $\begin{array}{l}\text { Late stage, } \\
\text { eyes }(\%)\end{array}$ \\
\hline Soft contact lens & $6(30.0)$ & $12(60.0)$ & $2(10.0)$ \\
Orthokeratology & $14(31.8)$ & $20(45.5)$ & $10(22.7)$ \\
\hline
\end{tabular}

the new baseline incidence of AK in the United States is 10 times greater than that before 2004 [14]. A study of AK among rigid gas-permeable contact lens wearers showed that nearly a quarter of patients were orthokeratology lens wearers from 2005 to 2011 [15]. In New Zealand, the incidence of AK in 2009 to 2016 had more than doubled when compared with the preceding 7-year period [4].

In previous studies, most cases related to contact lenses involved soft lenses $[4,6,16,17]$. However, more than two-thirds of AK cases were related to orthokeratology in this study. Even in Hong Kong, 37.5\% of contact lens-related AK was attributed to orthokeratology [18]. The reason may be that the myopic population in China has increased in recent years, and there has also been an increase in the average degree of myopia, especially among young children [19]. Orthokeratology has become one of the most popular corrective choices for parents. Consequently, many accompanying adverse effects associated with orthokeratology, such as AK, have been observed. Moreover, most orthokeratology users are children and teenagers; consequently, and the orthokeratology-associated AK patients were younger than the patients who used soft lenses. Therefore, it is necessary that patients and their parents learn about proper lens hygiene and care. Unlike other studies [4], the bilateral cases in this study were all associated with orthokeratology.

Early diagnosis is essential to ensure a good prognosis. Some studies have suggested that it is important to remember and consider AK in all cases of contact lens keratitis [20,21]. Clinical features of AK are similar to other types of keratitis, such as fungal and herpes simplex virus infections. Although confocal microscopy is a noninvasive technique [1], the images of the cysts were atypical in some of the early-stage cases [22]. The microbiological examination of corneal scrapings by microscopy is easy, fast and efficient. In this study, the positive rate of Giemsa-stained smears reached $84.4 \%$. In the early-stage cases, the rate was relatively low, while the positive rate of the $0.9 \% \mathrm{NaCl}$ wet mounts for the detection of trophozoites was slightly increased. Because drugs were not administered in some of the early-stage cases, encystment did not occur. Trophozoite activity can be observed directly by $0.9 \% \mathrm{NaCl}$ wet mounts. For highly suspected early-stage cases, especially those characterized by epitheliopathy, examinations with Giemsa-stained smears, $0.9 \% \mathrm{NaCl}$ wet mounts and confocal microscopy should be performed to help the early diagnosis of AK.

Culturing for Acanthamoeba was required for any patient suspected of having infective keratitis. The culturepositive rate of Acanthamoeba has been reported to be between 30 and $60 \%$ [20]. Even though culture requires 
a long incubation time, it is still the definitive diagnosis method [23]. In this study, the positive culture rate (76.6\%) was higher than that in previous reports, possibly because two-thirds of the cases were diagnosed in the advanced and late stages, and the corneal scrapings may have included more trophozoites and cysts.

It is notable that $\mathrm{AK}$ can cause coinfection with other organisms. It was reported that coinfections with bacteria were seen in 23\% of cases [24]. In a study in Egypt, bacteria were isolated from all the patients with AK [7]. In this study, $9.84 \%$ of the patients presented polymicrobial infection. This was similar to the result of our previous study (15\%) [25]. Because this survey was conducted in a tertiary referral centre, many cases received antibacterial treatment before anti-amoebic therapy. The result of the genotyping analysis was consistent with our previous study [9] and other studies in China [26]. Although the subtypes differ, genotype T4 is still the main AK-related genotype in China; furthermore, genotype T4 is the predominant genotype in other countries and areas [27-30].

To date, no drugs have been specifically approved for AK by the Food and Drug Administration [23]. Biguanides, including chlorhexidine and PHMB, are commonly used. In this study, more than two-thirds of the eyes were cured with medical therapy. Therapeutic penetrating keratoplasty is required for severe cases of $\mathrm{AK}$, but it is not recommended as a method for removal of the organisms from the cornea [20] because it is associated with poor outcomes, such as poor graft survival, repeat transplantation and glaucoma. Postoperative topical anti-amoeba drug therapy was also required for the patients in this study to avoid recurrence. In this study, the rate of therapeutic keratoplasty decreased after 2005 compared with the rate before 2005 in the orthokeratology group. There are several possible reasons. First, since 2003, orthokeratology has gradually entered into a state of relatively standard and healthy development in China [31]; additionally, clinicians gradually became highly suspicious of AK in keratitis related to contact lens use. Contact lens users were told to go to a hospital quickly if they had symptoms. Second, as confocal microscopy has high sensitivity and specificity in the diagnosis of AK, some cases were diagnosed rapidly. Third, as the number of cases increased, clinicians became more experienced in treatment.

The risk behaviours of orthokeratology lens and soft lens wearers were slightly different in this study. Approximately $87.8 \%$ of patients who used orthokeratology lenses rinsed their lenses with tap water, while the most common risk behaviours in SCL wearers were showering and sleeping while wearing their lenses. The risk behaviours were consistent with a survey in Egypt [7]. However, in the United States, storing lenses in tap water and topping off contact lens solution were the most common risk factors for $\mathrm{AK}$ in rigid gas-permeable contact lens wearers. Moreover, wearing lenses for orthokeratology overnight was also thought to be a risk factor [15]. Contact lens wearers, especially orthokeratology patients, should strictly adhere to good contact lens hygiene practices and avoid lens exposure to tap water to minimize the risk of AK.

There were some limitations to this study. As a retrospective study, some information, such as history, was incomplete during clinical data collection. Because this survey was conducted in one hospital, there might be bias in the percentage of cases associated with each lens type. More work is needed to explain the difference in the polymicrobial infection rate between orthokeratology lens and SCL wearers.

\section{Conclusions}

In conclusion, AK is one of the most challenging infections to manage. Although contact lens wear is not the most common risk factor for AK in China, AK cases related to contact lenses have been observed continuously over the years. More than two-thirds of cases were associated with orthokeratology lenses. The most common risk behaviour of orthokeratology users was rinsing lenses and/or cases with tap water, while in SCL users, it was wearing lenses while showering or sleeping. For highly suspected early-stage cases of, especially those characterized by epitheliopathy, examinations with Giemsa-stained smears, $0.9 \% \mathrm{NaCl}$ wet mounts and confocal microscopy should be performed to help the early diagnosis of AK. With increasing awareness of AK by clinicians, early diagnosis and effective medical treatment, the rate of therapeutic keratoplasty has decreased in recent years in orthokeratology users. Optometrists and ophthalmologists should teach contact lens wearers, especially orthokeratology users, to strictly adhere to good contact lens hygiene practices and avoid lens exposure to tap water to minimize the risk of AK.

\section{Additional file}

Additional file 1: Attachment 1 Cases of Acanthamoeba keratitis related to contact lens in each year (DOCX $13 \mathrm{~kb}$ )

\section{Abbreviations}

AK: Acanthamoeba keratitis; NaCl: Sodium chloride; SCL: Soft contact lens

\section{Acknowledgements}

The authors thank Dr. Yanlin Gao for her generous help with the comments and data analysis and Dr. Chao Jiang for assistance with the PCR assay.

\section{Authors' contributions}

WWL drafted the manuscript and performed the literature review. ZQW and YZ conducted the Acanthamoeba laboratory tests and cultures. JHQ participated in information gathering and editing. XGS conceived the idea and managed the patients' ophthalmic conditions. All authors read and approved the final manuscript. 


\section{Funding}

No funding was received by any of the authors for the writing of this manuscript.

Availability of data and materials

The data used to support the findings of this study are available from the corresponding author upon request.

\section{Ethics approval and consent to participate}

This study was performed in accordance with the Declaration of Helsinki and was approved by the Ethics Committee of Tongren Hospital, Beijing, China (approval number TRCKY-033). Informed consent was obtained from all the subjects after the aims and nature of the study were explained to the participants. Written informed consents were obtained from all patients participating in the investigation.

\section{Competing interests}

The authors declare that they have no competing interests.

\section{Author details}

${ }^{1}$ Tianjin Eye Hospital, Tianjin Ophthalmology and Visual Development Key Laboratory, Clinical College of Ophthalmology, Tianjin Medical University, Address: 4, Gansu Road, Heping District, Tianjin 300020, China. ${ }^{2}$ Beijing Institute of Ophthalmology, Beijing Tongren Eye Center, Beijing Tongren Hospital, Beijing Ophthalmology \& Visual Sciences Key Laboratory, Capital Medical University, Address: 17 Hou Gou Lane, Chong Nei Street, Beijing 100005, China.

Received: 12 May 2019 Accepted: 30 August 2019

Published online: 18 September 2019

\section{References}

1. Parmar DN, Awwad ST, Petroll WM, Bowman RW, McCulley JP, Cavanagh HD. Tandem scanning confocal corneal microscopy in the diagnosis of suspected acanthamoeba keratitis. Ophthalmology. 2006;113(4):538-47.

2. Dart JK, Saw VP, Kilvington S. Acanthamoeba keratitis: diagnosis and treatment update 2009. Am J Ophthalmol. 2009;148(4):487-99 e2.

3. Carnt N, Robaei D, Minassian DC, Dart JKG. Acanthamoeba keratitis in 194 patients: risk factors for bad outcomes and severe inflammatory complications. Br J Ophthalmol. 2018:102(10):1431-5.

4. McKelvie J, Alshiakhi M, Ziaei M, Patel DV, McGhee CN. The rising tide of Acanthamoeba keratitis in Auckland, New Zealand: a 7-year review of presentation, diagnosis and outcomes (2009-2016). Clin Exp Ophthalmol. 2018;46(6):600-7.

5. Cheung N, Nagra P, Hammersmith K. Emerging trends in contact lensrelated infections. Curr Opin Ophthalmol. 2016;27(4):327-32.

6. Dos Santos DL, Kwitko S, Marinho DR, de Araújo BS, Locatelli Cl, Rott MB. Acanthamoeba keratitis in Porto Alegre (southern Brazil): 28 cases and risk factors. Parasitol Res. 2018;117(3):747-50.

7. Taher EE, Méabed EMH, Abdallah I, Abdel Wahed WY. Acanthamoeba keratitis in noncompliant soft contact lenses users: genotyping and risk factors, a study from Cairo, Egypt. J Infect Public Health. 2018;11(3):377-83.

8. Jiang C, Sun $X$, Wang Z, Zhang Y. Acanthamoeba keratitis: clinical characteristics and management. Ocul Surf. 2015;13(2):164-8.

9. Zhang Y, Sun X, Wang Z, Li R, Luo S, Jin X, Deng S, Chen W. Identification of $18 \mathrm{~S}$ ribosomal DNA genotype of Acanthamoeba from patients with keratitis in North China. Invest Ophthalmol Vis Sci. 2004;45(6):1904-7.

10. Li W, Sun X, Wang Z, Zhang Y. A survey of contact lens-related complications in a tertiary hospital in China. Cont Lens Anterior Eye. 2018:41(2):201-4.

11. Xuguang S, Lin C, Yan Z, Zhiqun W, Ran L, Shiyun L, Xiuying J. Acanthamoeba keratitis as a complication of orthokeratology. Am J Ophthalmol. 2003;136(6):1159-61.

12. Sun $X$, Zhao H, Deng $S$, Zhang $Y$, Wang $Z$, Li R, Luo $S$, Jin $X$. Infectious keratitis related to orthokeratology. Ophthalmic Physiol Opt. 2006;26(2):133-6.

13. Watt KG, Swarbrick HA. Trends in microbial keratitis associated with orthokeratology. Eye Contact Lens. 2007;33(6 Pt 2):373-7 discussion 382.

14. Tu EY. Acanthamoeba keratitis: a new normal. Am J Ophthalmol. 2014; 158(3):417-9.
15. Cope JR, Collier SA, Schein OD, Brown AC, Verani JR, Gallen R, Beach MJ, Yoder JS. Acanthamoeba keratitis among rigid gas permeable contact Lens wearers in the United States, 2005 through 2011. Ophthalmology. 2016;123(7):1435-41.

16. Lindsay RG, Watters G, Johnson R, Ormonde SE, Snibson GR. Acanthamoeba keratitis and contact lens wear. Clin Exp Optom. 2007:90(5):351-60.

17. Patel A, Hammersmith K. Contact lens-related microbial keratitis: recent outbreaks. Curr Opin Ophthalmol. 2008;19(4):302-6.

18. Chan TC, Li EY, Wong WW, Jhanji V. Orthokeratology-associated infectious keratitis in a tertiary care eye hospital in Hong Kong. Am J Ophthalmol. 2014;158(6):1130-5 e2.

19. Liu YM, Xie P. The safety of orthokeratology--a systematic review. Eye Contact Lens. 2016;42(1):35-42.

20. Maycock NJ, Jayaswal R. Update on Acanthamoeba keratitis: diagnosis, treatment, and outcomes. Cornea. 2016:35(5):713-20

21. Lorenzo-Morales J, Khan NA, Walochnik J. An update on Acanthamoeba keratitis: diagnosis, pathogenesis and treatment. Parasite. 2015;22:10,

22. Kobayashi A, Yokogawa H, Yamazaki N, Ishibashi Y, Oikawa Y, Tokoro M, Sugiyama K. In vivo laser confocal microscopy findings of radial keratoneuritis in patients with early stage Acanthamoeba keratitis. Ophthalmology. 2013;120(7):1348-53.

23. Juárez MM, Tártara LI, Cid AG, Real JP, Bermúdez JM, Rajal VB, Palma SD. Acanthamoeba in the eye, can the parasite hide even more? Latest developments on the disease. Cont Lens Anterior Eye. 2018:41(3):245-51.

24. Sharma S, Garg P, Rao GN. Patient characteristics, diagnosis, and treatment of non-contact lens related Acanthamoeba keratitis. $\mathrm{Br} J$ Ophthalmol. 2000;84(10):1103-8.

25. Sun X, Zhang Y, Li R, Wang Z, Luo S, Gao M, Deng S, Chen W, Jin X Acanthamoeba keratitis: clinical characteristics and management. Ophthalmology. 2006;113(3):412-6.

26. Zhao G, Sun S, Zhao J, Xie L. Genotyping of Acanthamoeba isolates and clinical characteristics of patients with Acanthamoeba keratitis in China. J Med Microbiol. 2010:59(Pt 4):462-6.

27. Booton GC, Joslin CE, Shoff M, Tu EY, Kelly DJ, Fuerst PA. Genotypic identification of Acanthamoeba sp. isolates associated with an outbreak of acanthamoeba keratitis. Cornea. 2009;28(6):673-6.

28. Gatti S, Rama P, Matuska S, Berrilli F, Cavallero A, Carletti S, Bruno A, Maserati R, Di Cave D. Isolation and genotyping of Acanthamoeba strains from corneal infections in Italy. J Med Microbiol. 2010;59(Pt 11):1324-30.

29. Martín-Pérez T, Criado-Fornelio A, Martínez J, Blanco MA, Fuentes I, PérezSerrano J. Isolation and molecular characterization of Acanthamoeba from patients with keratitis in Spain. Eur J Protistol. 2017;61(Pt A):244-52.

30. Duarte JL, Furst C, Klisiowicz DR, Klassen G, Costa AO. Morphological, genotypic, and physiological characterization of Acanthamoeba isolates from keratitis patients and the domestic environment in Vitoria, Espírito Santo, Brazil. Exp Parasitol. 2013;135(1):9-14.

31. Xie P, Guo X. Chinese experiences on orthokeratology. Eye Contact Lens. 2016;42(1):43-7.

\section{Publisher's Note}

Springer Nature remains neutral with regard to jurisdictional claims in published maps and institutional affiliations.

Ready to submit your research? Choose BMC and benefit from:

- fast, convenient online submission

- thorough peer review by experienced researchers in your field

- rapid publication on acceptance

- support for research data, including large and complex data types

- gold Open Access which fosters wider collaboration and increased citations

- maximum visibility for your research: over $100 \mathrm{M}$ website views per year

At $\mathrm{BMC}$, research is always in progress.

Learn more biomedcentral.com/submissions 